\title{
Holographic Josephson junction from massive gravity
}

\author{
Ya-Peng $\mathrm{Hu},{ }^{1,5, *}$ Huai-Fan $\mathrm{Li}^{2, \dagger}$ Hua-Bi Zeng, ${ }^{3, \hbar}$ and Hai-Qing Zhang ${ }^{4, \S}$ \\ ${ }^{1}$ College of Science, Nanjing University of Aeronautics and Astronautics, Nanjing 210016, China \\ ${ }^{2}$ Institute of Theoretical Physics, Department of Physics, Shanxi Datong University, \\ Datong 037009, China \\ ${ }^{3}$ Department of Physics, National Central University, Chungli 320, Taiwan \\ ${ }^{4}$ Institute for Theoretical Physics, Utrecht University, Leuvenlaan 4, 3584 CE Utrecht, The Netherlands \\ ${ }^{5}$ State Key Laboratory of Theoretical Physics, Institute of Theoretical Physics, \\ Chinese Academy of Sciences, Beijing 100190, China \\ (Received 31 December 2015; published 3 May 2016)
}

\begin{abstract}
We study the holographic superconductor-normal metal-superconductor (SNS) Josephson junction in de Rham-Gabadadze-Tolley massive gravity. If the boundary theory is independent of spatial directions, i.e., if the chemical potential is homogeneous in spatial directions, we find that the graviton mass parameter will make it more difficult for the normal metal-superconductor phase transition to take place. In the holographic model of the Josephson junction, it is found that the maximal tunneling current will decrease according to the graviton mass parameter. Besides, the coherence length of the junction decreases as well with respect to the graviton mass parameter. If one interprets the graviton mass parameter as the effect of momentum dissipation in the boundary field theory, this indicates that the stronger the momentum dissipation is, the smaller the coherence length is.
\end{abstract}

DOI: 10.1103/PhysRevD.93.104009

\section{INTRODUCTION}

AdS/CFT correspondence [1-3] is a powerful tool to solve the strongly coupled physics from the one-dimensional higher, weakly coupled gravity in some limit, which is also dubbed "holography." In recent years, applied holography has attained great success in the area of condensed matter theory (CMT), hydrodynamics, etc. [4-6]. The holographic Josephson junction was first studied in [7] and was later largely extended to various models [8]. Other models of the holographic Josephson junction were proposed from designer multigravity in [9] and from D-branes in [10].

The gravity backgrounds in the above Josephson junctions are mostly Einstein's general relativity (GR). Due to the requirements of diffeomorphism invariance in GR, the corresponding graviton should be a massless spin-2 boson; see, for example, Ref. [11]. Therefore, it is a direct and interesting task in history to find whether there are other gravitational theories in which the graviton is massive [12]. However, the generalization is not so easy since usually the massive gravity has the instability problem of the Boulware-Deser ghost [13]. Recently, a nonlinear massive gravity theory has been proposed (the so-called dRGT theory by de Rham, Gabadadze, and Tolley) [14-16], and later it was found to be ghost-free $[17,18]$. For more details about the aspects of massive gravity, one can refer to the reviews $[16,19]$. Note that, during the proof of ghost-free

\footnotetext{
*huyp@nuaa.edu.cn

huaifan.li@stu.xjtu.edu.cn

zenghbi@gmail.com

${ }^{\S}$ H.Q.Zhang@uu.nl
}

dRGT massive gravity, the so-called reference metric $f_{\mu \nu}$ is assumed to be invertible. Also, dRGT massive gravity with a degenerated reference metric has also been widely investigated recently. For instance, the black hole solutions and their thermodynamics were studied in [20-25]. The counterterm of this massive gravity has also been obtained in [26]. It should be pointed out that, first, the dRGT massive gravity with a degenerated reference metric may bring about some healthy problems [27]. However, some works have shown that the ghost can still be free in this dRGT massive gravity with a degenerated reference metric $[20,23,28]$. In addition, by using this theory, the previous papers showed that the problems in this theory did not seem particularly pressing for a holographic theory (more arguments can also be seen in [27]). Second, dRGT massive gravity is thought to be the only healthy theory in Poincaré invariant setups. However, if one relaxes the condition of having the full Poincaré group, the number of available and healthy theories greatly increases $[29,30]$. Indeed, theories of massive gravity different from dRGT massive gravity have been considered in the holographic context in [31-33]. Third, in fact, a direct and significant motivation to investigate massive gravity comes from the holographic viewpoint. For example, the degenerated reference metric in dRGT massive gravity is usually chosen to break the diffeomorphism invariance of bulk spacetime in the spatial directions. Therefore, according to the AdS/CFT correspondence, the dual field theory in the boundary has a conserved energy but no conserved momentum currents, which can further correspond to the dissipation of momentum. In addition, a finite dc conductivity was obtained 
in $[20,27,34,35]$, while some other holographic results related to the effects of the graviton mass parameter in massive gravity have also been investigated in [21,36-39].

In this paper, we investigate the effect of the graviton mass parameter (or breaking of spatial translational symmetry in the boundary field theory due to AdS/CFT [27]) on the holographic Josephson junction in dRGT massive gravity with a degenerated reference metric. In particular, we study the superconductor-normal metal-superconductor (SNS) Josephson juction in the dRGT massive gravity. First, for a homogeneous superconductor, we find that the graviton mass parameter will reduce the critical temperature from a normal metal to a superconductor, which indicates that it is harder to have a phase transition from a normal metal to a superconductor when the graviton mass parameter is larger. Meanwhile, from the AdS/CFT aspect this also means that, in the boundary field theory, the larger the momentum dissipation is, the harder the phase transition is. This is reminiscent of the phase diagram of cuprates, where greater doping makes the phase transition from a normal metal to a superconductor more difficult. Although doping is not the same as the momentum dissipation, we can still argue that they have similar effects on the phase transitions from the point view of holography. For the holographic SNS Josephson junction, we find the usual sinusoidal relation between the tunneling current and the phase difference across the junction. One can obtain the maximal current by fitting the sinusoidal relation. It is found that the maximal current decreases exponentially with the width of the junction, from which we can get the coherence length of the normal metal in between the junction [40]. Moreover, we find that the coherence length decreases with respect to the graviton mass parameter, and so does the maximal current. Physically, this indicates that the momentum dissipation (or breaking of translational symmetry) will reduce the coherence length as well as the maximal current.

The paper is organized as follows. In Sec. II we briefly introduce dRGT massive gravity. We build up the holographic model of the Josephson junction in Sec. III. Numerical results are shown in Sec. IV, and conclusions are drawn in Sec. V. The dependence of the constraint equation with the gauge field equations is generically proven in Appendix.

\section{BRIEF REVIEW: DRGT MASSIVE GRAVITY AND ITS GENERAL BLACK HOLE SOLUTIONS}

In this paper, we focus on the ghost-free dRGT massive gravity, whose action in an $(n+2)$-dimensional spacetime usually reads as $[20,22]$

$$
S=\frac{1}{16 \pi G} \int d^{n+2} x \sqrt{-g}\left[R+\frac{n(n+1)}{L^{2}}+m^{2} \sum_{i}^{4} c_{i} \mathcal{U}_{i}(g, \mathfrak{\mathbf { f }})\right],
$$

where $\mathfrak{f}$ is a fixed symmetric tensor usually called the reference metric, $L$ is the radius of $\operatorname{AdS}_{n+2}$ spacetime, $c_{i}$ are constants, $m$ stands for the graviton mass parameter, ${ }^{1}$ and $\mathcal{U}_{i}$ are symmetric polynomials of the eigenvalues of the $(n+2) \times(n+2)$ matrix $\mathcal{K}^{\mu}{ }_{\nu} \equiv \sqrt{g^{\mu \alpha} \mathfrak{f}_{\alpha \nu}}$ :

$$
\begin{aligned}
& \mathcal{U}_{1}=[\mathcal{K}], \\
& \mathcal{U}_{2}=[\mathcal{K}]^{2}-\left[\mathcal{K}^{2}\right], \\
& \mathcal{U}_{3}=[\mathcal{K}]^{3}-3[\mathcal{K}]\left[\mathcal{K}^{2}\right]+2\left[\mathcal{K}^{3}\right], \\
& \mathcal{U}_{4}=[\mathcal{K}]^{4}-6\left[\mathcal{K}^{2}\right][\mathcal{K}]^{2}+8\left[\mathcal{K}^{3}\right][\mathcal{K}]+3\left[\mathcal{K}^{2}\right]^{2}-6\left[\mathcal{K}^{4}\right]
\end{aligned}
$$

The square root in $\mathcal{K}$ means $(\sqrt{A})^{\mu}{ }_{\nu}(\sqrt{A})^{\nu}{ }_{\lambda}=A^{\mu}{ }_{\lambda}$ and $[\mathcal{K}]=K^{\mu}{ }_{\mu}=\sqrt{g^{\mu \alpha} \mathfrak{f}_{\alpha \mu}}$. After making the variation of the action with respect to the metric, the equations of motion (EOM) become

$R_{\mu \nu}-\frac{1}{2} R g_{\mu \nu}-\frac{n(n+1)}{2 L^{2}} g_{\mu \nu}+m^{2} \chi_{\mu \nu}=8 \pi G T_{\mu \nu}$,

where

$$
\begin{aligned}
\chi_{\mu \nu}= & -\frac{c_{1}}{2}\left(\mathcal{U}_{1} g_{\mu \nu}-\mathcal{K}_{\mu \nu}\right)-\frac{c_{2}}{2}\left(\mathcal{U}_{2} g_{\mu \nu}-2 \mathcal{U}_{1} \mathcal{K}_{\mu \nu}+2 \mathcal{K}_{\mu \nu}^{2}\right) \\
& -\frac{c_{3}}{2}\left(\mathcal{U}_{3} g_{\mu \nu}-3 \mathcal{U}_{2} \mathcal{K}_{\mu \nu}+6 \mathcal{U}_{1} \mathcal{K}_{\mu \nu}^{2}-6 \mathcal{K}_{\mu \nu}^{3}\right) \\
& -\frac{c_{4}}{2}\left(\mathcal{U}_{4} g_{\mu \nu}-4 \mathcal{U}_{3} \mathcal{K}_{\mu \nu}+12 \mathcal{U}_{2} \mathcal{K}_{\mu \nu}^{2}\right. \\
& \left.-24 \mathcal{U}_{1} \mathcal{K}_{\mu \nu}^{3}+24 \mathcal{K}_{\mu \nu}^{4}\right) .
\end{aligned}
$$

Since the background we are going to use is $(3+1)$ dimensional, a general black hole solution can be [22]

$$
\begin{array}{r}
d s^{2}=-r^{2} f(r) d t^{2}+\frac{d r^{2}}{r^{2} f(r)}+r^{2} h_{i j} d x^{i} d x^{j}, \\
f(r)=\frac{k}{r^{2}}+\frac{1}{L^{2}}-\frac{m_{0}}{r^{3}}+\frac{q^{2}}{4 r^{4}}+\frac{c_{1} m^{2}}{2 r}+\frac{c_{2} m^{2}}{r^{2}}
\end{array}
$$

where $h_{i j} d x^{i} d x^{j}$ is the line element for the two-dimensional spherical, flat or hyperbolic space with $k=1,0$ or -1 , respectively. Here, $m_{0}$ is related to the mass of the black hole while $q$ is the charge of it. The reference metric can now be

$$
\mathfrak{f}_{\mu \nu}=\operatorname{diag}\left\{0,0, h_{i j}\right\} .
$$

The Hawking temperature of this black hole solution can be easily found,

\footnotetext{
${ }^{1}$ From [27] we know that the $m$ is related to the effective graviton mass which is perturbed around an "AdS-like" background; therefore, it is fair to call $m$ the graviton mass parameter.
} 


$$
\begin{aligned}
T_{\mathrm{BH}} & =\left.\frac{\left(r^{2} f(r)\right)^{\prime}}{4 \pi}\right|_{r=r_{+}} \\
& =\frac{1}{4 \pi r_{+}}\left(k+\frac{3 r_{+}^{2}}{L^{2}}-\frac{q^{2}}{4 r_{+}^{2}}+c_{1} m^{2} r_{+}+c_{2} m^{2}\right),
\end{aligned}
$$

in which $r_{+}$is the horizon of the black hole.

\section{HOLOGRAPHIC SETUP}

For simplicity, we consider the black hole solution in (2.5) with $k=0$ and $q=0$; therefore, $h_{i j}=\operatorname{diag}\left(h_{x x}, h_{y y}\right)=$ $\operatorname{diag}(1,1)$. In the probe limit, we adopt the Maxwell and complex scalar field action as

$$
S=\int d^{4} x \sqrt{-g}\left(-\frac{1}{4} F_{\mu \nu} F^{\mu \nu}-|\nabla \psi-i A \psi|^{2}-m_{\psi}^{2}|\psi|^{2}\right),
$$

in which $A_{\mu}$ is the $U(1)$ gauge field while $F_{\mu \nu}$ is the corresponding field strength with $F_{\mu \nu}=\partial_{\mu} A_{\nu}-\partial_{\nu} A_{\mu} ; m_{\psi}$ is the mass of the complex scalar field $\psi$. The EOMs can be obtained readily from the above action as

$$
\begin{gathered}
0=\left(\nabla_{\mu}-i A_{\mu}\right)\left(\nabla^{\mu}-i A^{\mu}\right) \psi-m_{\psi}^{2} \psi, \\
\nabla_{\nu} F^{\nu \mu}=i\left(\psi^{*}\left(\nabla^{\mu}-i A^{\mu}\right) \psi-\psi\left(\nabla^{\mu}+i A^{\mu}\right) \psi^{*}\right) .
\end{gathered}
$$

In order to work with the gauge-invariant fields, a natural ansatz for the fields is

$$
\begin{aligned}
\psi & =|\psi| e^{i \varphi}, \\
A_{\mu} & =\left(A_{t}, A_{r}, A_{x}, 0\right),
\end{aligned}
$$

where $|\psi|, \varphi, A_{t}, A_{r}, A_{x}$ are all real functions of $r$ and $x$. The corresponding gauge-invariant quantity can be defined as $M_{\mu} \equiv A_{\mu}-\partial_{\mu} \varphi$. Therefore, from the formulas (2.5), (3.2), (3.3) and (3.4), we obtain the following coupled partial differential equations (PDEs):

$$
\begin{aligned}
& \partial_{r}^{2}|\psi|+\frac{1}{r^{4} f} \partial_{x}^{2}|\psi|+\left(\frac{4}{r}+\frac{f^{\prime}}{f}\right) \partial_{r}|\psi| \\
& +\frac{1}{r^{2} f}\left(\frac{M_{t}^{2}}{r^{2} f}-r^{2} f M_{r}^{2}-\frac{M_{x}^{2}}{r^{2}}-L^{2} m_{\psi}^{2}\right)|\psi|=0, \\
& \partial_{r} M_{r}+\frac{1}{r^{4} f} \partial_{x} M_{x}+\frac{2}{|\psi|}\left(M_{r} \partial_{r}|\psi|+\frac{M_{x}}{r^{4} f} \partial_{x}|\psi|\right) \\
& +\left(\frac{4}{r}+\frac{f^{\prime}}{f}\right) M_{r}=0, \\
& \partial_{r}^{2} M_{t}+\frac{1}{r^{4} f} \partial_{x}^{2} M_{t}+\frac{2}{r} \partial_{r} M_{t}-\frac{2 L^{2}|\psi|^{2}}{r^{2} f} M_{t}=0, \\
& \partial_{x}^{2} M_{r}-\partial_{x} \partial_{r} M_{x}-2 L^{2} r^{2}|\psi|^{2} M_{r}=0,
\end{aligned}
$$

$$
\begin{gathered}
\partial_{r}^{2} M_{x}-\partial_{x} \partial_{r} M_{r}+\left(\frac{f^{\prime}}{f}+\frac{2}{r}\right)\left(\partial_{r} M_{x}-\partial_{x} M_{r}\right) \\
-\frac{2|\psi|^{2}}{L^{2} r^{2} f} M_{x}=0
\end{gathered}
$$

where ' denotes the derivative with respect to $r$. One can find that only gauge-invariant quantities are left in the above PDEs, and the phase $\varphi$ has been absorbed into the gauge-invariant quantity $M_{\mu}$. Moreover, there are only four independent EOMs in the above five EOMs since the second equation (3.5b) is just a constraint equation which results from the algebraic combinations of $(3.5 \mathrm{~d})$ and (3.5e) as ${ }^{2}$

$$
\begin{aligned}
\text { Eq. }(3.5 \mathrm{~b})= & -\frac{1}{2 r^{2}|\psi|^{2}}\left(\partial_{r}[\text { Eq. }(3.5 \mathrm{~d})]+\left(\frac{f^{\prime}}{f}+\frac{2}{r}\right)\right. \\
& \left.\times \text { Eq. }(3.5 \mathrm{~d})+\partial_{r}[\text { Eq. }(3.5 \mathrm{e})]\right) .
\end{aligned}
$$

Therefore, we correctly work with four independent EOMs for four fields, i.e., $|\psi|, M_{t}, M_{r}$ and $M_{x}$.

In order to solve the above coupled EOMs, we first need to impose boundary conditions for these fields. At the horizon, the field $M_{t}$ should vanish since $g^{t t}$ is divergent at the horizon, while other fields are finite at the horizon.

Near the infinite boundary $r \rightarrow \infty$, the fields $|\psi|, M_{r}$ and $M_{x}$ can be expanded as

$$
\begin{aligned}
|\psi|= & \frac{\psi^{(1)}(x)}{r^{\left(3-\sqrt{9+4 m_{\psi}^{2}}\right) / 2}}+\frac{\psi^{(2)}(x)}{r^{\left(3+\sqrt{9+4 m_{\psi}^{2}}\right) / 2}} \\
& +\mathcal{O}\left(\frac{1}{r^{\left(5+\sqrt{9+4 m_{\psi}^{2}}\right) / 2}}\right), \\
M_{t}= & \mu(x)-\frac{\rho(x)}{r}+\mathcal{O}\left(\frac{1}{r^{2}}\right), \\
M_{r}= & \frac{M_{r}^{(1)}(x)}{r^{2}}+\mathcal{O}\left(\frac{1}{r^{3}}\right), \\
M_{x}= & \nu(x)+\frac{J(x)}{r}+\mathcal{O}\left(\frac{1}{r^{2}}\right) .
\end{aligned}
$$

According to the AdS/CFT correspondence, the scalar field $|\psi|$ has conformal dimension $\Delta_{ \pm}=$ $\left(3 \pm \sqrt{9+4 m_{\psi}^{2}}\right) / 2$. In the following, we impose $\psi^{(1)} \equiv 0$, which indicates that there is no source term of the scalar operator on the boundary. According to the AdS/CFT dictionary, the coefficients $\psi^{(2)}, \mu, \rho, \nu$ and $J$ correspond to the condensate of the dual scalar operator $\langle\mathcal{O}\rangle$, chemical potential, charge density, superfluid velocity

\footnotetext{
${ }^{2}$ In the Appendix, we show how the constrained equation can be obtained from the Maxwell equation in a generic case.
} 
and current in the boundary field theory, respectively. The gauge-invariant phase difference $\gamma=\Delta \varphi-\int A_{x}$ across the weak link can be defined as [7]

$$
\gamma=-\int_{-\infty}^{+\infty} d x[\nu(x)-\nu( \pm \infty)]
$$

where $\nu( \pm \infty)$ is the superfluid velocity at spatial infinity $x= \pm \infty$.

In order to model a SNS Josephson junction, we choose the chemical potential $\mu(x)$ as

$$
\begin{aligned}
\mu(x)= & \mu_{\infty}\left\{1-\frac{1-\epsilon}{2 \tanh \left(\frac{\ell}{2 \sigma}\right)}\left[\tanh \left(\frac{x+\frac{\ell}{2}}{\sigma}\right)\right.\right. \\
& \left.\left.-\tanh \left(\frac{x-\frac{\ell}{2}}{\sigma}\right)\right]\right\},
\end{aligned}
$$

where $\mu_{\infty}=\mu(+\infty)=\mu(-\infty)$ is the chemical potential at $x= \pm \infty$, while $\ell, \sigma$ and $\epsilon$ are the width, steepness and depth of the junction, respectively. Following Ref. [7], we can define the critical temperature of the Josephson junction $T_{c}$ as the critical temperature of a homogeneous superconductor, i.e., with a flat chemical potential. Therefore, $T_{c}$ is proportional to $\mu_{\infty}=\mu( \pm \infty)$ :

$$
T_{c}=\frac{T_{\mathrm{BH}}}{\mu_{c}} \mu(\infty),
$$

where $\mu_{c}$ is the critical chemical potential of a homogeneous superconductor without any current at temperature $T_{\mathrm{BH}}$. Inside the junction, $x \in\left(-\frac{\ell}{2}, \frac{\ell}{2}\right)$, the effective critical temperature can be defined as

$$
T_{0}=\frac{T_{\mathrm{BH}}}{\mu_{c}} \mu(0) .
$$

Therefore, if one can set the profile of the chemical potential as $\mu(0)<\mu_{c}<\mu(\infty)$, from the relations (3.10) and (3.11) we know that $T_{0}<T_{\mathrm{BH}}<T_{c}$. Hence, the inbetween junction is in the normal metallic phase, while the region outside the junction is in the superconducting phase. In the following section, we will work in this way to model the SNS Josephson junction.

\section{NUMERICAL RESULTS}

There is a scaling symmetry in the PDEs (3.5):

$$
\begin{aligned}
& t \rightarrow \lambda t, \quad x \rightarrow \lambda x, \quad y \rightarrow \lambda y, \quad r \rightarrow \frac{1}{\lambda} r, \\
& M_{t} \rightarrow \frac{1}{\lambda} M_{t}, \quad M_{x} \rightarrow \frac{1}{\lambda} M_{x}, \quad M_{r} \rightarrow \lambda M_{r},
\end{aligned}
$$

where $\lambda$ is an arbitrary constant. We can adopt the above scaling symmetry (4.1) to set the horizon $r_{+}=1$ in the numerics. For convenience, we use the transformed coordinates in the following way: $u=1 / r$ and $y=\tanh \left(\frac{x}{4 \sigma}\right)$, as well as

$$
|\psi| \rightarrow \frac{|\psi|}{r^{\left(3-\sqrt{9+4 m_{\psi}^{2}}\right) / 2}}, \quad M_{r} \rightarrow \frac{M_{r}}{r^{2}} .
$$

Without loss of generality, we set the AdS radius $L=1$. We choose $m_{\psi}^{2}=-2$ in the numerics and the range of graviton mass parameters is $0 \leq m \leq 1.2$ since in the following we find that at $m \sim 1.2$ the maximal current is extremely close to zero as the width of the junction is large. For the convenience of numerics, we set $c_{1}=1, c_{2}=$ $-1 / 2$ in (2.6) in order to fix the horizon at $r_{+}=1$ by varying the mass of graviton $\mathrm{m}^{3}$ We solve the EOMs (3.5)-(3.5e) numerically by means of Chebyshev spectral methods [40].

\section{A. Critical temperature}

In this subsection, we study the phase diagram of the boundary theory with homogeneous chemical potential. The critical chemical potentials $\mu_{c}$ from normal metal states to superconductor states are from $\mu_{c} \approx 4.0638$ at $m=0$ to $\mu_{c} \approx 5.3306$ at $m=1.2$, which are shown in the left panel of Fig. 1. The phase diagram corresponding to the critical temperatures is plotted in the right panel of Fig. 1. The dark region is the normal metal phase, while the white region is the superconductor phase. On one hand, for a fixed graviton mass parameter $m$, lowering temperature (increasing chemical potential) will change the normal metal state to a superconductor state; on the other hand, for a fixed temperature (chemical potential), increasing graviton mass parameter $m$ will change the superconductor phase into a normal metal phase. The phase diagram in Fig. 1 is reminiscent of the famous phase diagram in the cuprates with doping, such as in Fig. 1 in [41]. Between the phases of superconductivity and Fermi liquid, greater doping will change a superconductor to a Fermi liquid or normal metal at a fixed temperature. Therefore, from this point of view there is a subtle relationship between the graviton mass parameter and the doping. We cannot make any definite conclusion about this relationship currently; however, they have a similar effect as the phase transition from superconductivity to a normal metal. A more complicated study of this phase transition has been done in [39], where the authors adopted a different action and metric from ours.

In order to model a SNS Josephson junction, from the above discussion we need to set $\mu(0)<\mu_{c}<\mu(\infty)$ for various $m$. After some trials, we find that a unified chemical potential $\mu(x)$ with the parameters $\mu_{\infty}=6, \sigma=0.7$ and $\epsilon=0.6$ will satisfy the requirements of the SNS junction.

\footnotetext{
${ }^{3}$ According to [22], the background in $(3+1)$ dimensions with $k=0$ is thermodynamically stable for $c_{2} \leq 0$.
} 

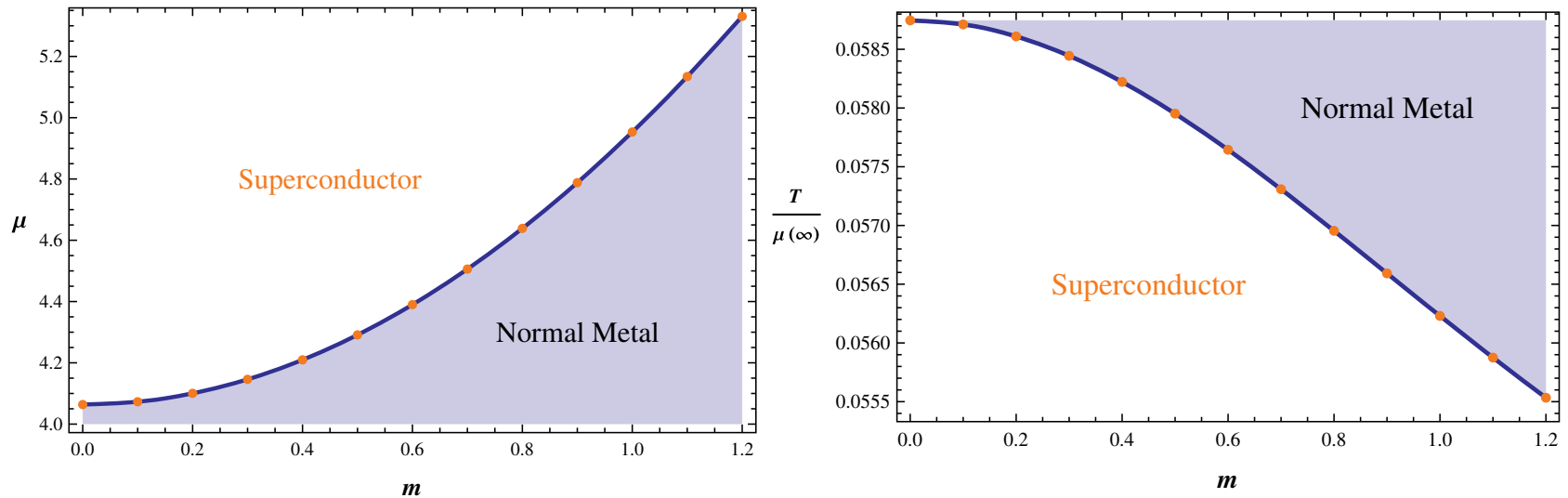

FIG. 1. Phase diagrams of a superconductor and normal metal. Left panel: Chemical potential versus graviton mass parameter $m$. Right panel: Temperature versus graviton mass parameter $m$. Orange dots are from numerical data.

We also choose $2 \leq \ell \leq 5$ in order to study the coherence length $\xi$ of the junction.

\section{B. Tunneling current}

Now we set the extra boundary conditions for the Josephson junctions. Close to the spatial boundary $x= \pm \infty$, we demand that all the fields are homogeneous, i.e., $\partial_{x}($ fields $)=0$. There is another symmetry of the fields when we flip the sign of $x \rightarrow-x$,

$$
\begin{aligned}
& |\psi| \rightarrow|\psi|, \quad M_{t} \rightarrow M_{t}, \quad M_{r} \rightarrow-M_{r}, \\
& M_{x} \rightarrow M_{x} .
\end{aligned}
$$

Therefore, $M_{r}$ is an odd function of $x$, while others are even. Thus, it is natural to set $M_{r}(x=0)=0$, while other fields have a vanishing first-order derivative with respect to $x$ at $x=0$. In the numerics, we set $J$ as a constant and make it an input parameter. Hence, the velocity $\nu$ and phase difference $\gamma$ can be obtained numerically. Moreover, we

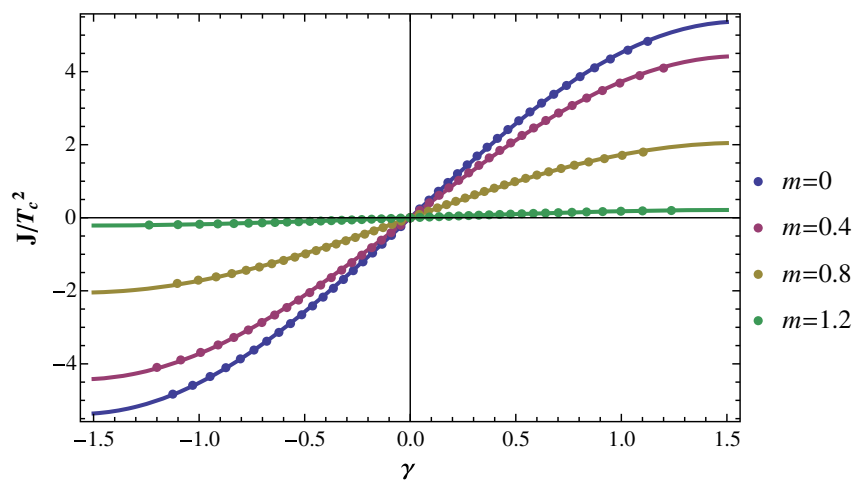

FIG. 2. The sinusoidal relation between the tunneling current $J / T_{c}^{2}$ and the phase difference $\gamma$ for various $m$. The width of the junction is now $\ell=2$. Dots are the numerical data, while the solid lines are the fittings of the data. find that it is convenient to work with dimensionless quantities, for instance, $J / T_{c}^{2}$.

When the junction width is $\ell=2$, we show the relation between the tunneling current $J / T_{c}^{2}$ and the phase difference of the junction $\gamma$ in Fig. 2. By using the sinusoidal relation $J \approx J_{\max } \sin (\gamma)$ to fit the data, we can figure out the maximal current $J_{\max }$ for each $m$ and $\ell$. In Fig. 3, we plot the relation between the maximal current $J_{\max }$ to the graviton mass parameter $m$ for various junction widths $\ell$. We find that for a fixed $\ell$, the maximal current will decrease as $m$ increases; meanwhile, for a fixed $m$, the maximal current also decreases when $\ell$ increases. Physically, this means increasing the graviton mass parameter (or, equivalently, increasing the momentum dissipation in the boundary) will suppress the tunneling between the two superconductors in both sides of the junction.

\section{Coherence length}

From condensed matter physics [40], there is a relation between the maximal current $J_{\max }$ and the coherence length $\xi$ as

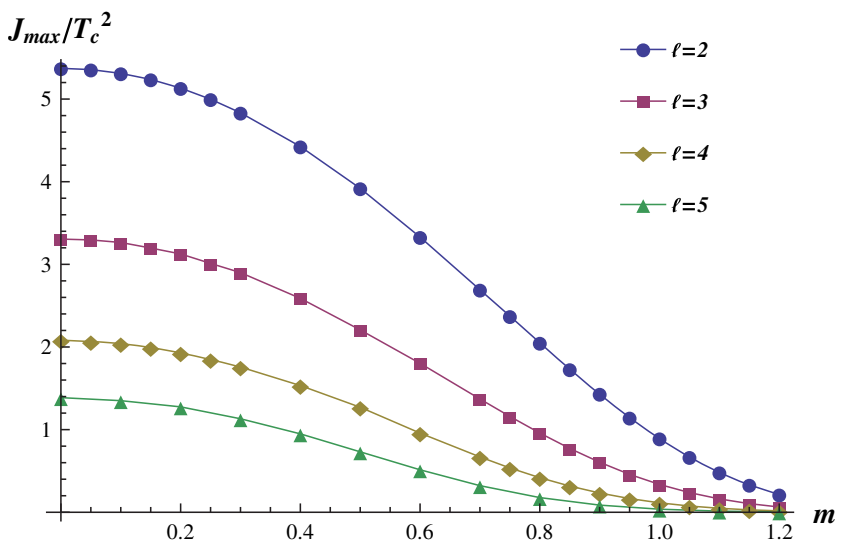

FIG. 3. Maximal current versus the graviton mass parameter for various junction widths $\ell$. 

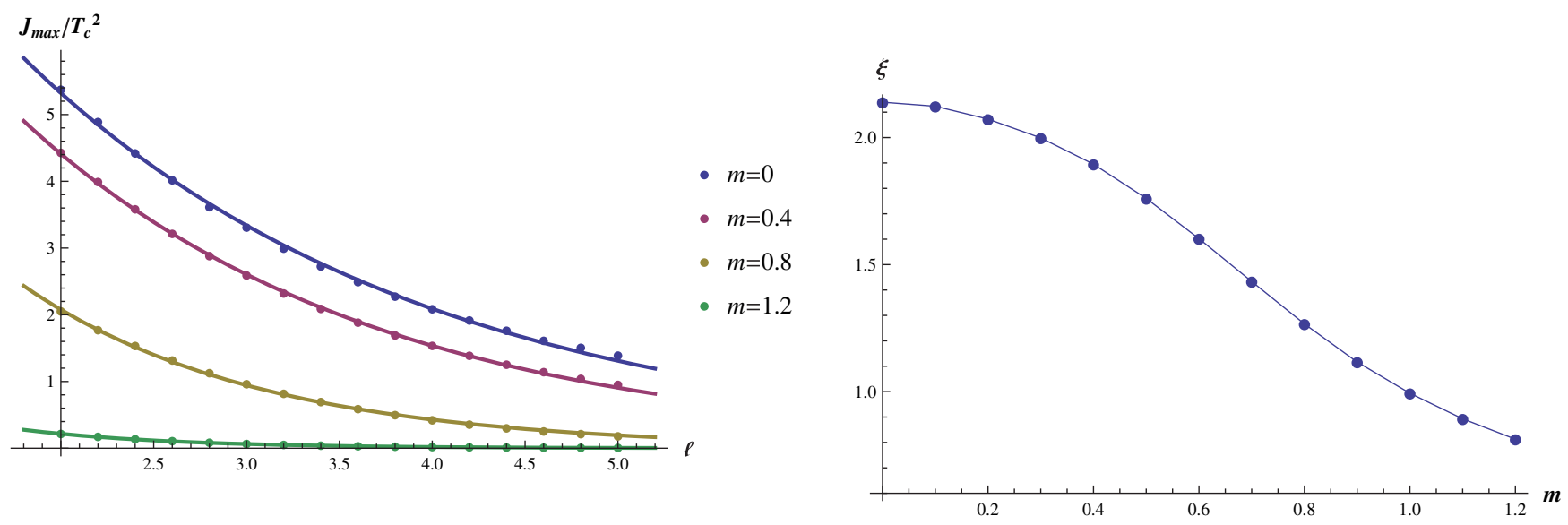

FIG. 4. Left panel: Maximum current to the width of the junction $\ell$ for various $m$. Right panel: Coherence length $\xi$ versus the graviton mass parameter $m$ from the fitting (4.4).

$$
J_{\max } / T_{c}^{2} \approx A e^{-\frac{\ell}{\xi}} .
$$

This relation holds when $\ell \gg \xi$, where $\xi$ is the coherence length of the normal metal. We show the numerics and fittings of the relation (4.4) in Fig. 4. From the left side of Fig. 4, we can see that for a fixed value of $m$, the maximal current has an exponential decay with respect to the width $\ell$. The fitted values of $\xi$ are shown on the right panel of Fig. 4. We can see that the coherence length will decrease as $m$ increases, which means that stronger momentum dissipation (breaking of translational symmetry) in the boundary field theory will reduce the coherence length $\xi$.

\section{CONCLUSIONS AND DISCUSSIONS}

In this paper, we studied the SNS Josephson junction in the background of dRGT massive gravity. For a homogeneous chemical potential, we found that the greater the graviton mass parameter, the more difficult for the normal metal-superconductor phase transition to occur. From the aspects of holography we argued that the phase transition would be more difficult if the momentum dissipation or breaking of translational symmetry was stronger in the boundary field theory. For the holographic SNS Josephson junction model, we obtained the familiar sinusoidal relation between the tunneling current and the phase difference across the junction. The maximal current would decrease by increasing the width of the junction, which was similar to what was found in previous studies. However, the more interesting result was that by increasing the graviton mass parameter in the bulk, the maximal current would decrease as well. Therefore, this indicated that stronger momentum dissipation would make the quantum tunneling in the Josephson junction more difficult. By virtue of the relation between the maximal current and the coherence length, we found that the coherence length would decrease as well with respect to the graviton mass parameter. Therefore, the momentum dissipation would also reduce the coherence length in the Josephson junction. We expect that this kind of relation between the maximal current, coherence length and the graviton mass parameter (momentum dissipation in the boundary field theory) can be observed in condensed matter experiments. It will also be interesting to find the analytic relation between the coherence length and the graviton mass parameter.

\section{ACKNOWLEDGMENTS}

We are grateful to KITPC for hospitality and partial support during the completion of this work. This work was partly supported by the National Natural Science Foundation of China (NSFC) under Grants No. 11105004, No. 11205097, No. 11575083, and No. 11565017; the Program for the Innovative Talents of Higher Learning Institutions of Shanxi; the Natural Science Foundation for Young Scientists of Shanxi Province, China (Grant No. 2012021003-4); the Fundamental Research Funds for the Central Universities under Grant No. NS2015073; Shanghai Key Laboratory of Particle Physics and Cosmology under Grant No. 11DZ2260700; The Open Project Program of State Key Laboratory of Theoretical Physics, Institute of Theoretical Physics, Chinese Academy of Sciences, China (No. Y5KF161CJ1).

\section{APPENDIX: GAUGE INVARIANCE AND CONSTRAINT EQUATION}

In this appendix, we show that for a generic Maxwellcomplex scalar action (3.1), the constraint equation obtained from the imaginary part of the complex scalar field equation can in fact be derived from the covariant derivative of the Maxwell equations. Therefore, the constraint equation actually is not independent from other equations. The key points lie behind the gauge invariance of the EOMs. Assuming gauge transformations (note that the fields $|\psi|, \varphi$ and $M_{\mu}$ are real functions), 


$$
\psi=|\psi| e^{i \varphi}, \quad A_{\mu}=M_{\mu}+\partial_{\mu} \varphi .
$$

The general complex scalar equation (3.2) becomes

$$
\left(\nabla_{\mu}-i M_{\mu}\right)\left(\nabla^{\mu}-i M^{\mu}\right)|\psi|-m_{\psi}^{2}|\psi|=0,
$$

and the general Maxwell equation (3.3) turns into

$$
\nabla_{\nu} F^{\nu \mu}=2 M^{\mu}|\psi|^{2} .
$$

Note that Eqs. (A2) and (A3) are now equations of only gauge-invariant quantities; the phase $\varphi$ disappears from the EOMs. Furthermore, Eq. (A2) can be decomposed into real and imaginary parts as

$$
\left(\nabla_{\mu} \nabla^{\mu}-M_{\mu} M^{\mu}-m_{\psi}^{2}\right)|\psi|-i\left(2 M^{\mu} \nabla_{\mu}+\left(\nabla_{\mu} M^{\mu}\right)\right)|\psi|=0 .
$$

The imaginary part of the complex scalar equation is actually the constraint equation [such as Eq. (3.5b)],

$$
2 M^{\mu} \nabla_{\mu}|\psi|+\left(\nabla_{\mu} M^{\mu}\right)|\psi|=0 .
$$

On the other hand, we can take the covariant derivative of the Maxwell equation (A3) and get [note that $\nabla_{\mu}\left(\nabla_{\nu} F^{\nu \mu}\right) \equiv$ 0 since $F^{\nu \mu}$ is antisymmetric in the indices]

$$
\begin{aligned}
0 \equiv & \nabla_{\mu}\left(\nabla_{\nu} F^{\nu \mu}\right) \\
= & 2 \nabla_{\mu}\left(M^{\mu}|\psi|^{2}\right) \\
& \Rightarrow\left(\nabla_{\mu} M^{\mu}\right)|\psi|+2 M^{\mu} \nabla_{\mu}|\psi|=0 .
\end{aligned}
$$

We can see that the right-hand side of Eq. (A6) is exactly the constraint equation (A5).
[1] J. M. Maldacena, The large N limit of superconformal field theories and supergravity, Int. J. Theor. Phys. 38, 1113 (1999) [Adv. Theor. Math. Phys. 2, 231 (1998)].

[2] S. S. Gubser, I. R. Klebanov, and A. M. Polyakov, Gauge theory correlators from noncritical string theory, Phys. Lett. B 428, 105 (1998).

[3] E. Witten, Anti-de Sitter space and holography, Adv. Theor. Math. Phys. 2, 253 (1998).

[4] S. A. Hartnoll, C. P. Herzog, and G. T. Horowitz, Building a Holographic Superconductor, Phys. Rev. Lett. 101, 031601 (2008).

[5] S. S. Lee, A Non-Fermi Liquid from a Charged Black Hole: A Critical Fermi Ball, Phys. Rev. D 79, 086006 (2009); H. Liu, J. McGreevy, and D. Vegh, Non-Fermi Liquids from Holography, Phys. Rev. D 83, 065029 (2011); M. Cubrovic, J. Zaanen, and K. Schalm, String theory, quantum phase transitions and the emergent Fermi-liquid, Science 325, 439 (2009).

[6] P. Kovtun, D. T. Son, and A. O. Starinets, Viscosity in Strongly Interacting Quantum Field Theories from Black Hole Physics, Phys. Rev. Lett. 94, 111601 (2005).

[7] G. T. Horowitz, J. E. Santos, and B. Way, A Holographic Josephson Junction, Phys. Rev. Lett. 106, 221601 (2011).

[8] Y. Q. Wang, Y.X. Liu, and Z.H. Zhao, Holographic Josephson junction in $3+1$ dimensions, arXiv:1104.4303; M. Siani, On inhomogeneous holographic superconductors, arXiv:1104.4463; Y. Q. Wang, Y.X. Liu, and Z. H. Zhao, Holographic p-wave Josephson junction, arXiv:1109.4426; Y. Q. Wang, Y.X. Liu, R. G. Cai, S. Takeuchi, and H. Q. Zhang, Holographic SIS Josephson junction, J. High Energy Phys. 09 (2012) 058; M. Rozali and A. Vincart-Emard, Chiral edge currents in a holographic Josephson junction, J. High Energy Phys. 01 (2014) 003; R. G. Cai, Y. Q. Wang, and H. Q. Zhang, A holographic model of SQUID, J. High
Energy Phys. 01 (2014) 039; S. Takeuchi, Holographic superconducting quantum interference device, Int. J. Mod. Phys. A 30, 1550040 (2015); H. F. Li, L. Li, Y. Q. Wang, and H. Q. Zhang, Non-relativistic Josephson junction from holography, J. High Energy Phys. 12 (2014) 099; S. Liu and Y. Q. Wang, Holographic model of hybrid and coexisting s-wave and p-wave Josephson junction, Eur. Phys. J. C 75, 493 (2015).

[9] E. Kiritsis and V. Niarchos, Josephson junctions and AdS/CFT networks, J. High Energy Phys. 07 (2011) 112 [10 (2011) 95].

[10] S. K. Domokos, C. Hoyos, and J. Sonnenschein, Holographic Josephson junctions and Berry holonomy from D-branes, J. High Energy Phys. 10 (2012) 073.

[11] A. Zee, Einstein Gravityina Nutshell (Princeton University Press, Princeton, NJ, 2013).

[12] M. Fierz and W. Pauli, On relativistic wave equations for particles of arbitrary spin in an electromagnetic field, Proc. R. Soc. A 173, 211 (1939).

[13] D. G. Boulware and S. Deser, Can Gravitation Have a Finite Range?, Phys. Rev. D 6, 3368 (1972).

[14] C. de Rham and G. Gabadadze, Generalization of the FierzPauli action, Phys. Rev. D 82, 044020 (2010).

[15] C. de Rham, G. Gabadadze, and A. J. Tolley, Resummation of Massive Gravity, Phys. Rev. Lett. 106, 231101 (2011).

[16] K. Hinterbichler, Theoretical aspects of massive gravity, Rev. Mod. Phys. 84, 671 (2012).

[17] S. F. Hassan and R. A. Rosen, Resolving the Ghost Problem in Non-Linear Massive Gravity, Phys. Rev. Lett. 108, 041101 (2012).

[18] S. F. Hassan, R. A. Rosen, and A. Schmidt-May, Ghost-free massive gravity with a general reference metric, J. High Energy Phys. 02 (2012) 026. 
[19] C. de Rham, Massive gravity, Living Rev. Relativity 17, 7 (2014).

[20] D. Vegh, Holography without translational symmetry, arXiv: 1301.0537.

[21] A. Adams, D. A. Roberts, and O. Saremi, Hawking-Page Transition in Holographic Massive Gravity, Phys. Rev. D 91, 046003 (2015).

[22] R. G. Cai, Y. P. Hu, Q. Y. Pan, and Y. L. Zhang, Thermodynamics of Black Holes in Massive Gravity, Phys. Rev. D 91, 024032 (2015).

[23] Y. P. Hu and H. Zhang, Misner-Sharp Mass and the Unified First Law in Massive Gravity, Phys. Rev. D 92, 024006 (2015).

[24] J. Xu, L. M. Cao, and Y. P. Hu, P-V Criticality in the Extended Phase Space of Black Holes in Massive Gravity, Phys. Rev. D 91, 124033 (2015).

[25] S. H. Hendi, B. E. Panah, and S. Panahiyan, Einstein-BornInfeld-massive gravity: AdS-black hole solutions and their thermodynamical properties, J. High Energy Phys. 11 (2015) 157; Thermodynamical structure of AdS black holes in massive gravity with stringy gauge-gravity corrections, arXiv: 1510.00108.

[26] L. M. Cao and Y. Peng, Counterterms in massive gravity theory, arXiv:1509.08738; L. M. Cao, Y. Peng, and Y. L. Zhang, On dRGT Massive Gravity with Degenerate Reference Metrics, arXiv:1511.04967 [Phys. Rev. D (to be published)].

[27] M. Blake and D. Tong, Universal Resistivity from Holographic Massive Gravity, Phys. Rev. D 88, 106004 (2013).

[28] H. Zhang and X. Z. Li, Ghost Free Massive Gravity with Singular Reference Metrics, arXiv:1510.03204 [Phys. Rev. D (to be published)].

[29] S. L. Dubovsky, Phases of massive gravity, J. High Energy Phys. 10 (2004) 076.

[30] V. A. Rubakov and P. G. Tinyakov, Infrared-modified gravities and massive gravitons, Phys. Usp. 51, 759 (2008).
[31] T. Andrade and B. Withers, A simple holographic model of momentum relaxation, J. High Energy Phys. 05 (2014) 101.

[32] M. Baggioli and O. Pujolas, Electron-Phonon Interactions, Metal-Insulator Transitions, and Holographic Massive Gravity, Phys. Rev. Lett. 114, 251602 (2015).

[33] L. Alberte, M. Baggioli, A. Khmelnitsky, and O. Pujolas, Solid holography and massive gravity, J. High Energy Phys. 02 (2016) 114.

[34] R. A. Davison, Momentum Relaxation in Holographic Massive Gravity, Phys. Rev. D 88, 086003 (2013).

[35] M. Blake, D. Tong, and D. Vegh, Holographic Lattices Give the Graviton an Effective Mass, Phys. Rev. Lett. 112, 071602 (2014).

[36] R. A. Davison, K. Schalm, and J. Zaanen, Holographic duality and the resistivity of strange metals, Phys. Rev. B 89, 245116 (2014).

[37] A. Amoretti, A. Braggio, N. Maggiore, N. Magnoli, and D. Musso, Thermo-electric transport in gauge/gravity models with momentum dissipation, J. High Energy Phys. 09 (2014) 160; A. Amoretti, A. Braggio, N. Maggiore, N. Magnoli, and D. Musso, Analytic dc Thermoelectric Conductivities in Holography with Massive Gravitons, Phys. Rev. D 91, 025002 (2015); A. Amoretti, A. Braggio, N. Magnoli, and D. Musso, Bounds on intrinsic diffusivities in momentum dissipating holography, J. High Energy Phys. 07 (2015) 102.

[38] H. B. Zeng and J. P. Wu, Holographic Superconductors from the Massive Gravity, Phys. Rev. D 90, 046001 (2014).

[39] M. Baggioli and M. Goykhman, Phases of holographic superconductors with broken translational symmetry, J. High Energy Phys. 07 (2015) 035; Under the dome: Doped holographic superconductors with broken translational symmetry, J. High Energy Phys. 01 (2016) 011.

[40] M. Tinkham, Introduction to Superconductivity (McGrawHill, New York, 1975).

[41] C. Varma, High-temperature superconductivity: Mind the pseudogap, Nature (London) 468, 184 (2010). 